\title{
Therapeutic properties of isoliquiritigenin with molecular modeling studies: investigation of anti- pancreatic acinar cell tumor and HMG-CoA reductase inhibitor activity for treatment of hypercholesterolemia
}

Jihua $\mathrm{Li}^{1}$, Fengfeng Zhur ${ }^{2}$, Weiguo $\mathrm{Xu}^{1}$, Ping $\mathrm{Che}^{3}$

\author{
${ }^{1}$ Interventional Radiology Department of Zhuhai Hospital affiliated with Jinan \\ University (Zhuhai People's Hospital), Zhuhai, Guangdong, China \\ ${ }^{2}$ Department of Hepatobiliary Surgery, the First Affiliated Hospital of University \\ of South China, Hengyang, Hunan, China \\ ${ }^{3}$ Department of General Surgery, Chongqing Hechuan District Maternal and Child \\ Health Care Hospital, Chongqing, China
}

Submitted: 22 December 2021; Accepted: 31 December 2021

Online publication: 15 January 2021

Arch Med Sci

DOI: https://doi.org/10.5114/aoms/145448

Copyright $\odot 2022$ Termedia \& Banach

\section{Abstract}

Introduction: Isoliquiritigenin, one of the components in the root of Glycyrrhiza glabra L., is a member of the flavonoids, which are known to have anti-tumor activity in vitro and in vivo. HMG-CoA reductase inhibitors, called statins, are used to reduce the risk of heart disease by lowering blood cholesterol levels.

Material and methods: HMG-CoA reductase activity was determined according to the method described by Takahashi et al. The structure of human HMG-COA reductase in the resolution of $2.22 \AA$ with the X-ray diffraction method (PDB ID: 1 HWK) was obtained from the PDB database.

Results: In our study, the inhibitory activity of isoliquiritigenin towards HMG-CoA reductase showed a lower value of $\mathrm{IC}_{50}=193.77 \pm 14.85 \mu \mathrm{g} / \mathrm{ml}$. For a better understanding of biological activities and interactions, the molecular docking study was performed. The results of molecular docking revealed that isoliquiritigenin with a docking score of -6.740 has a strong binding affinity to HMG-COA reductase. Therefore, this compound could be considered as a potential inhibitor for the enzyme. Also, the activity of isoliquiritigenin against common human pancreatic acinar cell tumor cell lines, i.e. 266-6, TGP49, and TGP47, was evaluated.

Conclusions: The cells treated with isoliquiritigenin were assessed by MTT assay for $48 \mathrm{~h}$ as regards the cytotoxicity and anti-human pancreatic acinar cell tumor properties in normal (HUVEC) and human pancreatic acinar cell tumor cell lines, i.e. 266-6, TGP49, and TGP47. The IC ${ }_{50}$ values of isoliquiritigenin were 262,389 , and $211 \mu \mathrm{g} / \mathrm{ml}$ against 266-6, TGP49, and TGP47 cell lines, respectively. The viability of the human pancreatic acinar cell tumor cell line decreased dose-dependently in the presence of isoliquiritigenin. After clinical study, isoliquiritigenin can be utilized as an efficient drug in the treatment of human pancreatic acinar cell tumor in humans.

Key words: isoliquiritigenin, HMG-COA reductase, human pancreatic acinar cell tumor, molecular docking.

\author{
Corresponding author: \\ Ping Che \\ Department of \\ General Surgery \\ Chongqing Hechuan \\ District Maternal and \\ Child Health \\ Care Hospital \\ Chongqing, China \\ E-mail: \\ cheping77777@sina.com
}




\section{Introduction}

The compound isoliquiritigenin (ISL) is one of the biological active ingredients isolated from the roots of plants belonging to licorice, including Mongolian glycyrrhiza, Glycyrrhiza glabra, Glycyrrhizauralensis, and so forth. Licorice is used in alternative medicine and common foods, and its derivative, ISL, is applied for disease treatment e.g. cancer therapy, food additives and antibiotic therapy, and so on. The isoliquiritigenin compound can be isolated from the roots of Glycyrrhiza and was considered to be the main biologically active component for its useful pharmacological properties such as anti-viral, anti-microbial, anticancer activities, anti-oxidative, anti-inflammatory, hepatoprotective, immunomodulatory, and cardioprotective effects [1, 2].

The enzyme 3-hydroxy-3-methylglutaryl coenzyme A (HMG-CoA) reductase catalyzes the conversion of HMG-CoA to mevalonate, a four-electron oxidoreduction, which is the rate limiting step in the synthesis of cholesterol and other isoprenoids. The enzyme is found in eukaryotes and prokaryotes; and phylogenetic analysis revealed two classes of HMG-CoA reductase, Class I enzymes of eukaryotes, and Class II enzymes of some archaea and eubacteria and some other archaea [3]. The three-dimensional structures of the catalytic domain of HMG-CoA reductases obtained from humans and the bacterium Pseudomonas mevalonii revealed details of the catalysis mechanism in connection with site-directed mutagenesis studies [4]. The reaction catalyzed by human HMG-CoA reductase is a target for anti-hypercholesterolemic drugs (statins) aimed at lowering serum cholesterol levels. 3-hydroxy-3-methyl-glutaryl coenzyme A (HMG-CoA) reductase inhibitors are agents with strong blood cholesterol and lipid lowering effects and are used in primary and secondary prevention in atherosclerosis. However, studies have shown that its mortality-lowering effects in atherosclerosis cannot be explained by its lipid-lowering effects alone. Therefore, these agents have been shown to have anti-inflammatory, immunomodulatory, endothelial dysfunction correcting effects, as well as procoagulant activity and platelet functions $[5,6]$.

In addition to experimental investigation of biological materials such as enzymes and their related inhibitors, theoretical studies could also provide valuable data as complementary information. One of the characteristics of theoretical studies is providing an adequate insight into the interactions and inhibition mechanisms of various ligands in the presence of enzymes [7]. Although there are a variety of theoretical methods, molecular docking studies have attracted considerable attention in this area. The outcomes obtained from molecular docking calculations can support the results of the experimental section. There could be different interactions and activities for a chemical compound in the presence of an enzyme, and these activities are predictable by molecular docking studies [8].

We also investigated isoliquiritigenin in cytotoxicity studies against common human anti-human pancreatic acinar cell tumor in vitro. Interestingly, we obtained significantly good results in the study. Also, we performed enzyme inhibition and molecular docking studies.

\section{Material and methods}

\section{Enzyme study}

HMG-CoA reductase activity according to the method described by Takahashi et al.; [9] (DL$\mathrm{HMG}-\mathrm{CoA}+2 \mathrm{NADPH}+2 \mathrm{H}^{+} \rightarrow(\mathrm{R})$-mevalonate + $2 \mathrm{NADP}^{++}$was measured spectrophotometrically using the absorbance change of NADPH at $340 \mathrm{~nm}$ in accordance with the reaction. After zero adjustment against distilled water at $340 \mathrm{~nm}$, phosphate buffer was added to the test tubes separately as a blank and sample for each sample. $\mathrm{NaN}_{3}, \mathrm{NADPH}$ and the sample were added and mixed thoroughly and the absorbance change was followed for 1 min [10]. Then HMG-CoA was added to the cuvette and the absorbance change was monitored for 3 min. HMG CoA activity was calculated using the coefficient of NADPH. $\left(\varepsilon: 6.22 \times 10^{3} \mathrm{l} / \mathrm{mol} \times \mathrm{cm}\right)$ is given as IU/I activities are measured, then we started the $\mathrm{IC}_{50}$ study after the control value was found [11]. For the $I C_{50}$ study, we used six different inhibitor concentrations and wrote down the activities and then plotted the $I C_{50}$. After obtaining the (concentration-percent activity) equation, we calculated the $\mathrm{IC}_{50}$ value. We repeated this task 3 times and calculated the standard deviation and found that our work was correct [12].

\section{Molecular docking study}

The structure of human HMG-COA reductase in the resolution of $2.22 \AA$ with the $\mathrm{X}$-ray diffraction method (PDB ID: $1 \mathrm{HWK}$ ) [13] was obtained from the PDB database (http://www.rcsb.org/pdb). The enzyme structure was prepared for computational studies using the protein preparation module of the Schrödinger Suite [14]. For the preparation, the hydrogen atoms were added to the structure, and the water molecules were removed beyond $5 \AA$. The structure was optimized for the creation of an $\mathrm{H}$-bond network, and finally, the system was subjected to minimization using the OPLS3e force field. After the minimization step, the prepared protein was assessed for prediction of the binding sites using SiteMap of Schrödinger [15]. The Receptor Grid Generation module was then utilized 
to create a grid box of $20 \times 20 \times 20\left(\AA^{3}\right)$ around the first active site. The structure of isoliquiritigenin as an SDF file was downloaded from the PubChem database and was prepared with the LigPrep module [16]. The correct molecular geometries were generated, and suitable protonation states were produced using this module. After the preparation of the receptor and ligand, the molecular docking calculations were conducted using Glide.

\section{Anti-human pancreatic acinar cell tumor properties of isoliquiritigenin}

Cell culture at a much lower cost and time answers many questions and on the other hand prevents the indiscriminate killing of laboratory animals. This means that the cells extracted from that organism can be used for various studies and there is no need for direct testing on that organism. Cell culture has given rise to model systems. In other words, if there is a need for molecular study of specific tissue cells of an organism, by holding the cells of that tissue, the study can be performed. Today, many intracellular chemical processes, cellular and molecular defects that cause disease, the effects of different drugs on cells, topics related to aging, as well as cell-based products in cell culture, can be investigated. Cancer cell culture is one of the most basic applications of cell culture studies in the field of cancer. Cancer cells are immortal and can be cultured for long periods. Therefore, the existence of different cell lines with an unlimited lifespan has created a system for conducting studies in this field. Drugs recommended for the treatment of cancer must first be examined to see which cancer pathways are effective. Toxicity tests also provide ample space to study the effects of various drugs on cell survival. Sometimes the goal is to kill cancer cells, and the effect of the drug is seen in terms of its effect on different pathways of death. Sometimes a drug is produced for other diseases with specific purposes that must be proven to not affect cell survival. Today, a large number of animals also die as a result of testing the effects of health and cosmetics produced; cell culture has been able to cover many of these tests to prevent the indiscriminate killing of animals in related industries. Cell culture, in addition to creating an unlimited living laboratory space for drug testing, has been able to help humans in the field of cell therapy with the development of three-dimensional culture and tissue engineering. Today, much of the discussion of tissue production and genetic engineering for gene therapy is done through cell culture. People with disabilities can regain lost tissue by receiving engineered stem cells. Although this hypothesis is not complete today, it is not far from the truth, because it is estimated that by 2040 it will be a significant part of the world's revenue from tissue engineering. Also in people who are born with a genetic defect, with full knowledge of the defect of the genetic code through cell culture, they can be treated. Cell culture has created a suitable environment to treat diseases in vitro by biotechnology techniques through trial and error and, after obtaining appropriate results, it has been generalized to humans [17].

In this study, the MTT (Sigma Aldrich, Germany) colorimetric method was used to investigate the cell death effects of isoliquiritigenin on human pancreatic acinar cell tumor cell lines. Concentrations of $0-1000 \mu \mathrm{g} / \mathrm{ml}$ of isoliquiritigenin were treated in 266-6, TGP49, and TGP47 cell lines for $24 \mathrm{~h}$. After this time, the contents of the 96-well plate wells were carefully removed and MTT (Microculture Tetrazolium Test) dye was added to it. Then, it was kept at $37{ }^{\circ} \mathrm{C}$ for $4 \mathrm{~h}$ under $5 \%$ carbon dioxide with $90 \%$ humidity. The MTT dye was then isolated and the formazan crystals produced by the living cells were dissolved in isopropanol. Finally, the sample absorption was read using an ELISA reader (ELISA reader, Oraganon Teknika, Netherlands) at a wavelength of $570 \mathrm{~nm}$ and the cell viability rate was calculated by the following formula [17]: Cell viability (\%) $=($ Sample A/Control A) $\times 100$.

Then, based on the absorption rate of each well and its comparison with the control, the inhibitory concentration of $50 \%\left(\mathrm{IC}_{50}\right)$ was obtained [17].

\section{Statistical analysis}

All data obtained from this study were the result of three replications of three independent experiments. Data were statistically analyzed using Microsoft Excel 2019 software and Student's $t$-test. Data with a value of $p<0.05$ were considered statistically significant.

\section{Results and Discussion}

\section{Enzyme results}

The anti-inflammatory properties of HMG-CoA reductase inhibitors are explained by many different mechanisms. Statins support NO release and prevent leukocyte-epithelium interaction. The increase in NO release suppresses the infiltration of leukocytes, especially neutrophils, into the inflamed area. Statins prevent the activation of $T$ cells and the accumulation of monocytes and T cells into the arterial wall [18]. It has also been shown that the expression of adhesion molecules (P-selectin and intercellular adhesion molecule-1 (ICAM-1)) and cytokines (interleukin 6 and 8 (IL-6, IL-8)) from endothelial cells is suppressed. With the use of statins, the levels of proinflammatory cytokines (TNF- $\alpha, \mathrm{IL}-1 \beta)$ are decreased. Sim- 
ilarly, cerivastatin, an HMG-CoA reductase inhibitor, has been shown to inhibit ICAM-1 synthesis in response to lipopolysaccharide from endothelial cells [19]. Apart from that, HMG-CoA reductase inhibitors also reduce the release of various chemoattractant molecules (protein-1 (MCP-1) and IL-8) from monocytes by acting on nuclear factor $\kappa B$ (NF-KB). HMG-CoA reductase inhibitors also inhibit CRP synthesis. CRP is an acute phase reactant; it is synthesized in the liver in response to proinflammatory cytokines, and it impairs endothelial functions by reducing eNOS expression in endothelial cells. HMG-CoA reductase inhibitors also have antithrombotic properties [20]. During inflammation, thrombomodulin expression decreases on the endothelial surface and tissue factor expression increases; this situation creates a tendency to thrombosis. The use of HMG-CoA reductase inhibitors increases thrombomodulin expression and decreases tissue factor synthesis and activity. In our study, inhibitory activity of isoliquiritigenin towards HMG-CoA reductase showed a lower value of $\mathrm{IC}_{50}=193.77 \pm 14.85 \mu \mathrm{g} / \mathrm{ml}$.

\section{Analysis of anti-human pancreatic acinar cell tumor effects of isoliquiritigenin}

Regarding the cytotoxicity of antioxidant molecules, several studies have been performed and it has been determined that the toxicity of antioxidant molecules is greater than that of non-antioxidant molecules. However, the effects of antioxidant molecules on human health or the environment in general are still debated and need further research and study. Studies have also shown that antioxidant molecules can be effective at the cellular, microcellular, and molecular levels, such as proteins and genes. Evaluation of antioxidant molecules in cellular and animal models indicates that these particles cause cytotoxicity by inducing the production of passive oxygen species. In this regard, a decrease in the amount of glutathione, an increase in the amount of passive oxygen and an increase in lipid peroxidation have been reported in samples treated with metal antioxidant molecules. According to previous research, antioxidant molecules have toxic activity towards cancerous cells, which may be because of the tumor cells' relatively acidic environment in which the release of metal ions from antioxidant molecules occurs more rapidly. In addition, the metal toxic effects on cancer cells could be because of the interaction of metal atoms with functional groups of proteins, as well as phosphate and nitrogen bases of the DNA molecule. Another effect of antioxidant molecules on cancer cells is growth inhibition by inhibiting cell division, which results in their interaction with the structure and orga- nization of the actin cytoskeleton. Evidence and results of several studies indicate the induction of apoptosis as a result of the use of antioxidant molecules in cancer cells. This seems to happen through the production of reactive oxygen. Thus, the active surfaces of antioxidant molecules yield free radicals by releasing metal ions, and these free radicals destroy DNA, especially the mitochondrial membrane, causing the release of cytochrome $c$ and the induction of apoptosis from the mitochondrial pathway [17].

In recent years, researchers have reported that biological molecules have unique anti-human cancer effects. Biological molecules have received remarkable attention in the field of medicine [17] Several reports conducted recently have revealed that some biological molecules have remedial activities and it is a unique alternative to antibacterial and especially anticancer drugs. Biological molecules as a particular kind of well-known molecules have been applied recently to treat various cancers and tumors.

In this study, the cells treated with different concentrations of isoliquiritigenin were assessed by MTT assay for $48 \mathrm{~h}$ as regards the cytotoxicity properties in normal (HUVEC) and human pancreatic acinar cell tumor cell lines, i.e. 266-6, TGP49, and TGP47 (Figures 1-4).

The absorbance rate was evaluated at $570 \mathrm{~nm}$, which represented viability on the normal cell line (HUVEC) even up to $1000 \mu \mathrm{g} / \mathrm{ml}$ for isoliquiritigenin (Table I and Figure 4). The viability of the pancreatic acinar cell tumor cell line decreased dose-dependently in the presence of isoliquiritigenin. The $\mathrm{IC}_{50}$ values of isoliquiritigenin were 262, 389, and $211 \mu \mathrm{g} / \mathrm{ml}$ against 266-6, TGP49, and TGP47 cell lines, respectively (Table I).

\section{Molecular docking results}

The biological activities and interactions of isoliquiritigenin were assessed using molecular docking as a versatile theoretical approach. The docking pose of this compound among residues of the HMG-COA reductase is presented in Figure 5 . The probable interactions of isoliquiritigenin with HMG-COA reductase are shown in Figure 6. As can be seen, the compound has created six hydrogen bonds with Thr558, Met657, Asn658, Met659, and Gly765. Most of these amino acids are among the residues introduced as catalytic residues [21]. Asn658 has created two hydrogen bonds, which shows the high activity of this residue. On the other hand, as it is apparent, there are two active oxygens in the structure of isoliquiritigenin. Each one of these oxygens has constructed two hydrogen bonds with residues of the enzyme. One of these oxygens has $\mathrm{H}$-bonds with $\mathrm{NH}$ of the peptide backbone of residues Met657 and Asn658. 


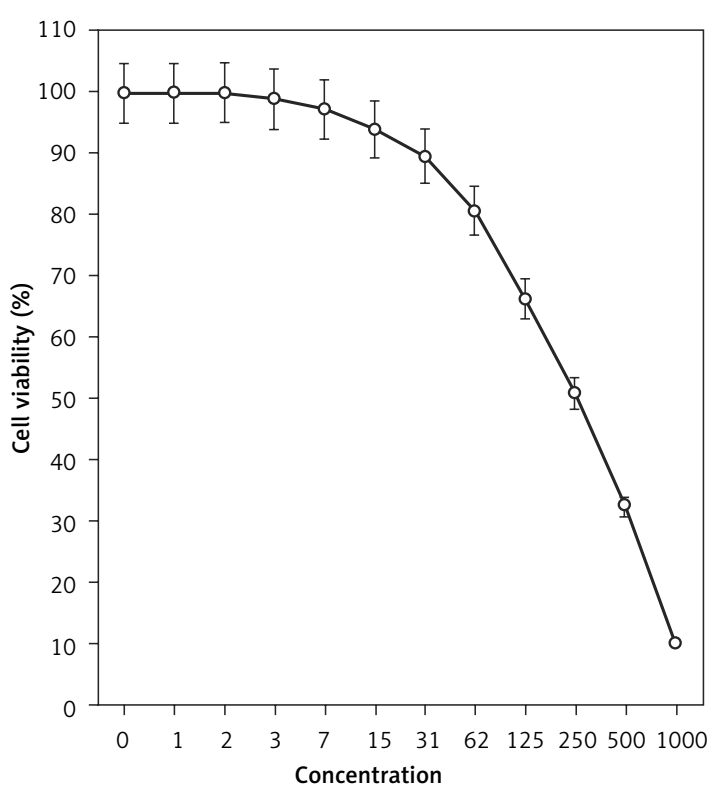

Figure 1. Anti-pancreatic acinar cell tumor effects of isoliquiritigenin (concentrations of $0-1000 \mu \mathrm{g} / \mathrm{ml}$ ) against 266-6 cell line

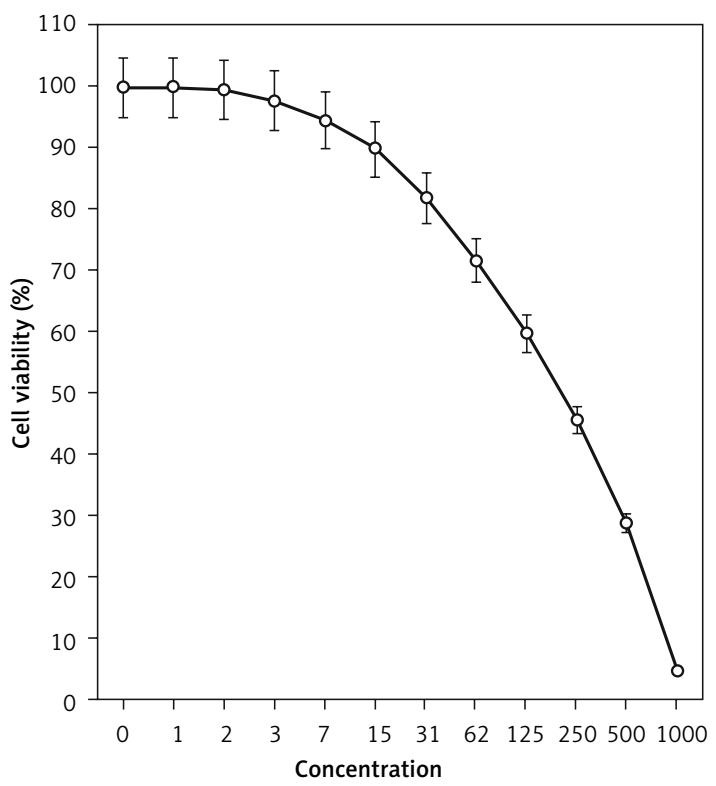

Figure 3. Anti-pancreatic acinar cell tumor effects of isoliquiritigenin (concentrations of $0-1000 \mu \mathrm{g} / \mathrm{ml}$ ) against TGP47 cell line

The other active oxygen has made $\mathrm{H}$-bonds with the oxygen of the peptide backbone of residues Thr558 and Gly765. As Glu559 is one of the reported catalytic residues [21]; the $\mathrm{H}$-bond of Thr558 is a crucial hydrogen bond because this is the nearest amino acid to Glu559. There are also eight hydrophobic contacts. These interactions have led to a desirable binding affinity of isoliquiritigenin, which makes this compound a potential inhibitor for HMG-COA reductase. Glu559 and Asp767 are previously reported to be members of the catalytic site of the enzyme [21]. Based on the

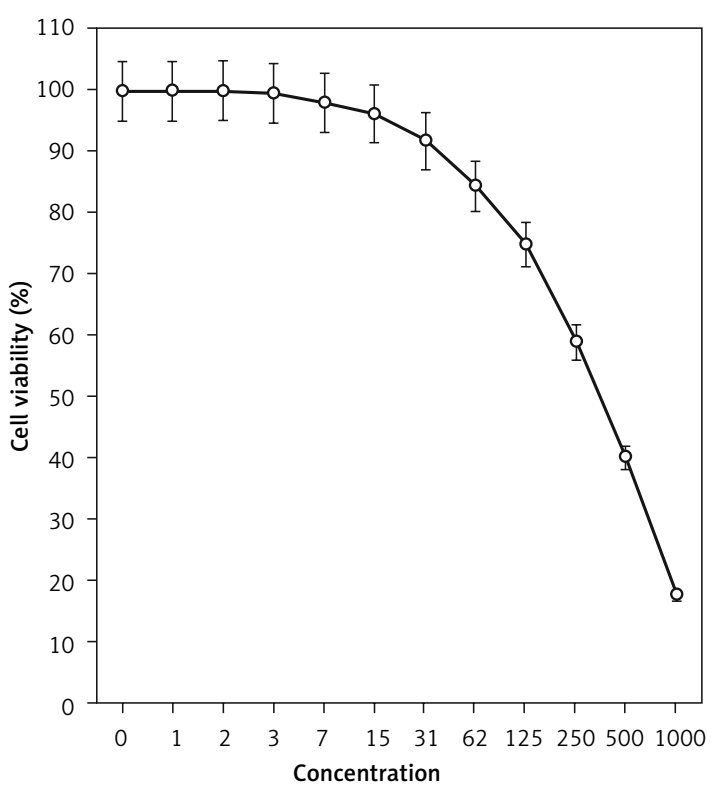

Figure 2. Anti-pancreatic acinar cell tumor effects of isoliquiritigenin (concentrations of $0-1000 \mu \mathrm{g} / \mathrm{ml}$ ) against TGP49 cell line.

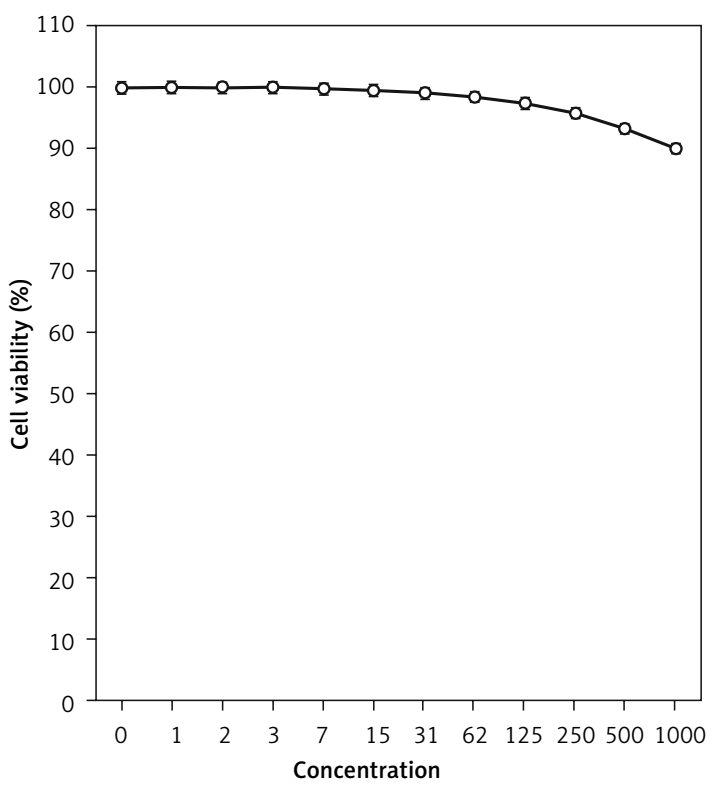

Figure 4. Cytotoxicity activities of isoliquiritigenin against normal (HUVEC) cell line

Table I. The $\mathrm{IC}_{50}$ of isoliquiritigenin in the anti-pancreatic acinar cell tumor experiment

\begin{tabular}{|lc|}
\hline & Isoliquiritigenin $[\mathrm{\mu g} / \mathrm{ml}]$ \\
\hline$I C_{50}$ against HUVEC & - \\
\hline$I C_{50}$ against $266-6$ & $262 \pm 0^{\mathrm{a}}$ \\
\hline$I C_{50}$ against TGP49 & $389 \pm 0^{\mathrm{b}}$ \\
\hline$I \mathrm{C}_{50}$ against TGP47 & $211 \pm 0^{\mathrm{a}}$ \\
\hline
\end{tabular}

obtained interactions in Figure 6, isoliquiritigenin has created a hydrophobic contact and a hydrogen bond with Asp 767 and Thr558. Considering 


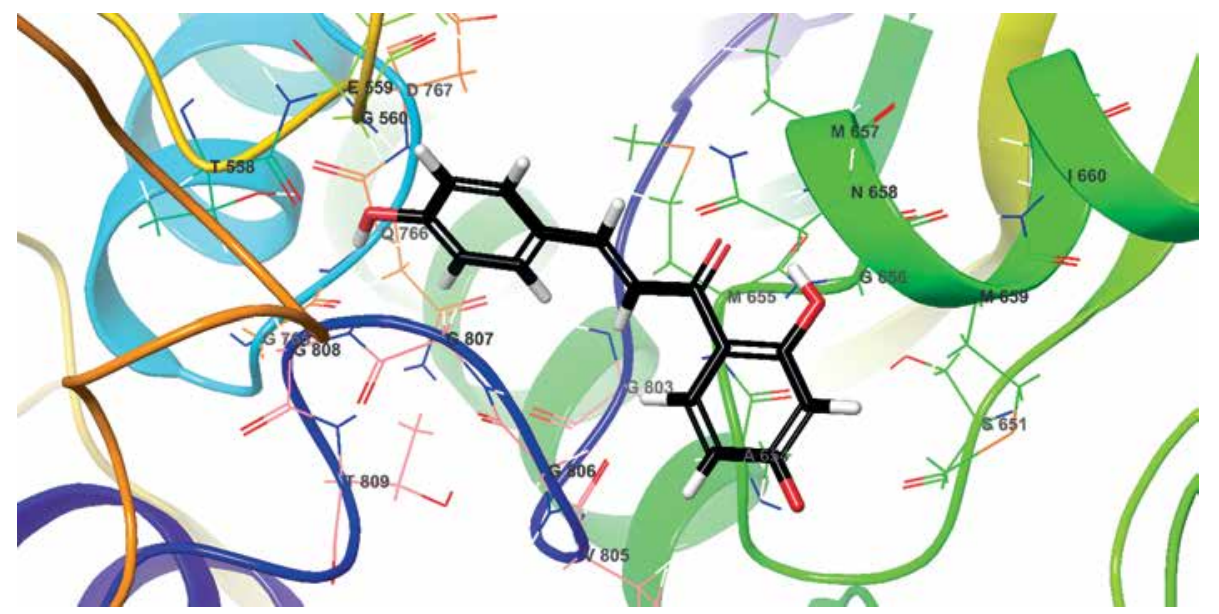

Figure 5. The docking pose of isoliquiritigenin among HMG-COA reductase

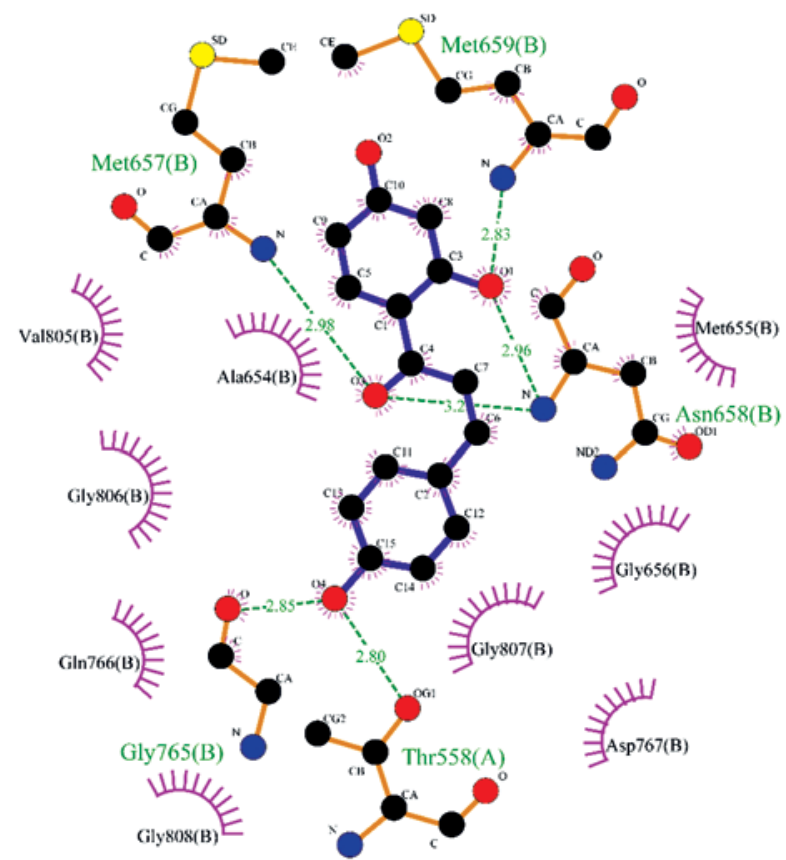

Figure 6. Interactions of isoliquiritigenin and HMG-COA reductase. Green dashed lines indicate hydrogen bonds, and semicircles show hydrophobic contacts

Table II. Parameters obtained from the molecular docking calculations

\begin{tabular}{|lc|}
\hline Parameter & $\begin{array}{l}\text { HMG-CoA } \\
\text { reductase }\end{array}$ \\
\hline $\mathrm{IC}_{50}[\mathrm{mM}]$ & 193.77 \\
\hline Docking score $[\mathrm{kcal} / \mathrm{mol}]$ & -6.740 \\
\hline Glide ligand efficiency $[\mathrm{kcal} / \mathrm{mol}]$ & -0.355 \\
\hline Glide Ecoul $[\mathrm{kcal} / \mathrm{mol}]$ & -10.851 \\
\hline Glide Evdw $[\mathrm{kcal} / \mathrm{mol}]$ & -26.474 \\
\hline Glide Emodel $[\mathrm{kcal} / \mathrm{mol}]$ & -46.318 \\
\hline Glide energy $[\mathrm{kcal} / \mathrm{mol}]$ & -37.325 \\
\hline
\end{tabular}

the previously reported catalytic residues, this compound has the ability to bind tightly to the active residues. This binding affinity indicates that isoliquiritigenin could be considered as a potential inhibitor for HMG-COA reductase. Some of the essential parameters obtained from the docking calculations are shown in Table II. The docking score is the most important calculated value [22], which indicates the binding affinity between the structures. The Glide Ligand Efficiency is another parameter that is numerically obtained and shows the efficiency of the molecule. Some other parameters such as Glide Evdw and Glide Ecoul are interaction related parameters. The energy of the interaction is numerically calculated (Glide energy), 


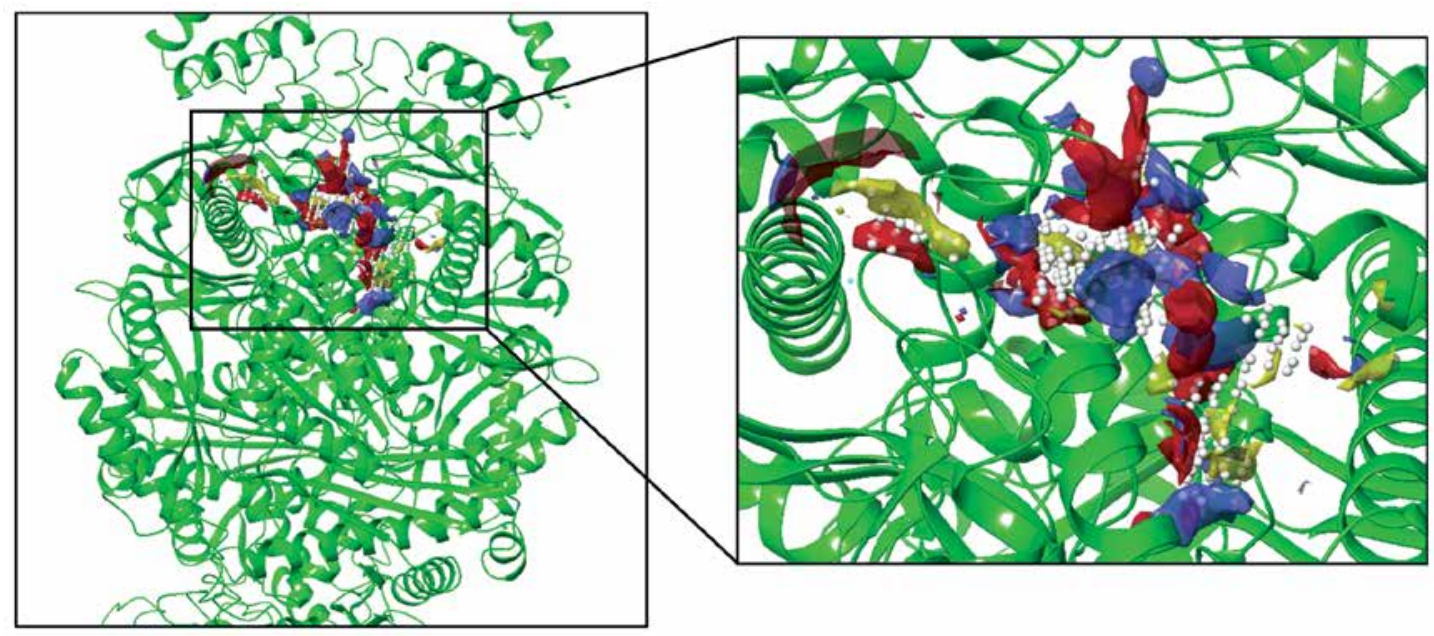

Figure 7. The first active site in the HMG-COA reductase structure

Table III. Residues of the first active site with Dscore of 1.040 and site score of 1.072

\begin{tabular}{|lr|}
\hline Chain & Predicted residues of the first active site \\
\hline A & Residues: $536,556,557,558,559,560,561,562,565,735,751,752,755,758,759,762,853,857$ \\
\hline B & Residues: $525,526,590,628,652,653,654,655,656,657,658,659,683,684,686,688,689,690,691,692,735,7$ \\
& $62,765,766,767,768,769,770,801,802,803,805,806,807,808,809,810,814,826,831$ \\
\hline
\end{tabular}

and the value of the interaction pose is presented with Glide Emodel [23]. The prediction of enzyme active sites was accomplished with the SiteMap module of Schrödinger. The first active site with a Dscore of 1.04 was chosen as the catalytic site of the enzyme. The position of this active site in the enzyme structure is presented in Figure 7 , and the residues of this site are presented in Table III. The essential factor for a predicted active site is the Dscore greater than 1, which makes this site a druggable site. Due to the potential druggability of the first active site, it could be considered as the catalytic active site. The characteristics of the predicted active site, and the obtained results for ligand-enzyme interactions, approve the docking pose of the isoliquiritigenin among enzyme residues. The outcomes of docking calculations will also be supported consequently.

In conclusion, the molecular docking study can provide adequate information about the interactions and chemical activities of compounds in the presence of biological materials such as enzymes. The results of the docking study revealed the strong binding affinity of isoliquiritigenin to HMG-COA reductase. Based on this computational investigation, isoliquiritigenin is a potential inhibitor for this enzyme.

The viability of pancreatic acinar cell tumor cell lines decreased dose-dependently in the presence of isoliquiritigenin. The $\mathrm{IC}_{50}$ values of isoliquiritigenin were 262,389 , and $211 \mu \mathrm{g} / \mathrm{ml}$ against 266-6, TGP49, and TGP47 cell lines, respectively. It seems that the anti-human pancreatic acinar cell tumor effect of this molecule is due to its antioxidant effects.

\section{Acknowledments}

Jihua Li and Fengfeng Zhu are co-first authors, they contributed equally to this work.

\section{Conflict of interest}

The authors declare no conflict of interest.

\section{References}

1. Chen H, Zhang B, Yuan X, et al. Isoliquiritigenin-induced effects on Nrf2 mediated antioxidant defence in the HL-60 cell monocytic differentiation. Cell Biol Int 2013; 37: 1215-24.

2. Chen X, Wu Y, Jiang Y, et al. Isoliquiritigenin inhibits the growth of multiple myeloma via blocking IL-6 signaling. J Mol Med 2012; 90: 1311-9.

3. Bochar DA, Stauffacher CV, Rodwell VW. Sequence comparisons reveal two classes of 3-hydroxy-3-methylglutaryl coenzyme A reductase. Mol Genet Metab 1999; 66: 122-7.

4. Hampton RY, Rine J. Regulated degradation of HMGCoA reductase, an integral membrane protein of the endoplasmic reticulum, in yeast. J Cell Biol 1994; 125: 299-312.

5. Hampton R, Dimster-Denk D, Rine J. The biology of HMG-CoA reductase: the pros of contra-regulation. Trends Biochem Sci 1996; 21: 140-5.

6. Burg JS, Powell DW, Chai R, Hughes AL, Link AJ, Espenshade PJ. Insig regulates HMG-CoA reductase by controlling enzyme phosphorylation in fission yeast. Cell Metab 2008; 8: 522-31. 
7. Koçyiğit ÜM, Taslimi P, Tüzün $B$, Yakan $H$, Muğlu $H$, Güzel E. 1,2,3-Triazole substituted phthalocyanine metal complexes as potential inhibitors for anticholinesterase and antidiabetic enzymes with molecular docking studies. J Biomol Struct Dyn 2020; doi: 10.1080/07391102.2020.1857842.

8. Jhong CH, Riyaphan J, Lin SH, Chia YC, Weng CF. Screening alpha-glucosidase and alpha-amylase inhibitors from natural compounds by molecular docking in silico. BioFactors 2015; 41: 242-51.

9. Takahashi M, Kusumi K, Shumiya S, Nagase S. Plasma lipid concentrations and enzyme activitiesin Nagase Analbuminemia Rat (NAR). Exp Animals 1983; 32: 39-46.

10. Suzuki H, Aoki T, Tamaki T, Sato F, Kitahara M, Saito $Y$ Hypolipidemic effect of NK-104, a potent HMG-CoA reductase inhibitor, in guinea pigs. Atherosclerosis 1999; 146: 259-70.

11. Kumar A, Sharma N, Gupta A, Kalonia H, Mishra J. Neuroprotective potential of atorvastatin and simvastatin (HMG-CoA reductase inhibitors) against 6-hydroxydopamine (6-OHDA) induced Parkinson-like symptoms. Brain Res 2012; 1471: 13-22.

12. Alvi SS, Iqbal D, Ahmad S, Khan MS. Molecular rationale delineating the role of lycopene as a potent HMG-CoA reductase inhibitor: in vitro and. In silico study. Nat Prod Res 2016; 30: 2111-4.

13. Istvan ES, Deisenhofer J. Structural mechanism for statin inhibition of HMG-CoA reductase. Science 2001; 292: 1160-4

14. "Schrödinger Release 2020-4: Protein Preparation Wizard; Epik, Schrödinger, LLC, New York, NY 2016; Impact, Schrödinger, LLC, New York, NY 2016; Prime, Schrödinger, LLC, New York, NY 2020.

15. Poustforoosh A, Hashemipour H, Tüzün B, Pardakhty A Mehrabani M, Nematollahi MH. Evaluation of potential anti-RNA-dependent RNA polymerase (RdRP) drugs against the newly emerged model of COVID-19 RdRP using computational methods. Biophys Chem 2021; 272: 106564.

16. “Schrödinger Release 2020-4: LigPrep, Schrödinger, LLC, New York, NY 2020

17. Lu Y, Wan X, Li L, Sun P, Liu G. Synthesis of a reusable composite of graphene and silver nanoparticles for catalytic reduction of 4- nitrophenol and performance as anti-colorectal carcinoma. J Materials Res Technol 2021 12: $1832-43$

18. Nabi R, Alvi SS, Saeed M, Ahmad S, Khan MS. Glycation and HMG-CoA reductase inhibitors: implication in diabetes and associated complications. Curr Diabetes Rev 2019; 15: 213-23.

19. Ressaissi A, Attia N, Falé PL, et al. Isorhamnetin derivatives and piscidic acid for hypercholesterolemia: cholesterol permeability, HMG-CoA reductase inhibition, and docking studies. Arch Pharmacol Res 2017; 40: 1278-86.

20. Iqbal D, Khan MS, Khan MS, Ahmad S, Hussain MS, Ali M. Bioactivity guided fractionation and hypolipidemic property of a novel HMG-CoA reductase inhibitor from Ficus virens Ait. Lipids Health Dis 2015; 14: 15.

21. Haines BE, Wiest O, Stauffacher CV. The increasingly complex mechanism of HMG-CoA reductase. Acc Chem Res 2013; 46: 2416-26.

22. Subhani S, Jayaraman A, Jamil K. Homology modelling and molecular docking of MDR1 with chemotherapeutic agents in non-small cell lung cancer. Biomed Pharmacother 2015; 71: 37-45.

23. Türe A, Kahraman DC, Cetin-Atalay R, Helvacıoğlu S, Charehsaz M, Küçükgüzel İ. Synthesis, anticancer activ- ity, toxicity evaluation and molecular docking studies of novel phenylaminopyrimidine-(thio)urea hybrids as potential kinase inhibitors. Comput Biol Chem 2019; 78: $227-41$ 